Contreras, O. E., Stein R., \& Vecino, C. E. (2014). Diseño y evaluación retrospectiva de una estrategia de inversión en el mercado bursátil colombiano mediante la maximización del ratio de Sharpe. Revista Lebret (6). Bucaramanga, Colombia: Universidad Santo Tomás, pp. 303-320. ISSN 2145-5996.

\title{
Diseño y evaluación retrospectiva de una estrategia de inversión en el mercado bursátil colombiano mediante la maximización del ratio de Sharpe*
}

\section{Design and retrospective evaluation of an investment strategy in the colombian stock exchange market, through the maximization of the Sharpe's Ratio}

\author{
Orlando E. Contreras \\ Roberto Stein Bronfman ${ }^{2}$ \\ Carlos Enrique Vecino
}

\begin{abstract}
Resumen
El artículo presenta el informe de los resultados obtenidos por la aplicación de un modelo de optimización desarrollado mediante el uso del ratio de Sharpe, que en un análisis retrospectivo simula una estrategia de inversión, teniendo en cuenta los datos históricos del mercado bursátil colombiano. De su aplicación, se obtienen portafolios anuales recomendados que se comparan con los valores reales del Indice de la Bolsa de Valores de Colombia (IGBC). Los resultados alcanzados dan cuenta de la efectividad del modelo formulado en las condiciones planteadas, toda vez que sus recomendaciones demuestran un desempeño superior al del mercado. Los aportes de este estudio apuntan hacia el potencial de profundización de su análisis para otros escenarios y en la eventual posibilidad de adopción del modelo en decisiones reales de inversión.
\end{abstract}

\section{Palabras clave}

Portafolios de inversión, mercado bursátil, análisis retrospectivo, ratio de Sharpe.

\section{Códigos de clasificación JEL: G11, G12, G24}

\begin{abstract}
This current article represents the briefing of the results obtained by applying a optimization model developed through the use of Sharpe's Ratio, which through a retrospective analysis simulates an investment strategy taking into account historic data of the Colombian Stock Exchange Market. From its application, annual recommended portfolios are obtained, then they are compared with the real Colombia Stock Exchange Market values (IGBC).The obtained results give evidence of the effectiveness of the formulated model in the established conditions, since their recommendations show a higher performance in the market. The contributions of this study aim at the deepening potential for other scenarios and the eventual possibility of adopting the model in real investment situations.
\end{abstract}

\section{Keywords}

investment portfolio, stock exchange market, retrospective analysis, Sharpe's Ratio

Este artículo se deriva del proyecto de investigación denominado "Relación rentabilidad-riesgo en inversiones", del grupo de investigación Finance \& Management, de la Escuela de Estudios Industriales y Empresariales, de la Universidad Industrial de Santander, Bucaramanga, Colombia.

1 Magíster en Administración de Empresas. Profesor asistente de la Escuela de Estudios Industriales y Empresariales, Universidad Industrial de Santander, Bucaramanga, Colombia. Correo electrónico: ocontrer@ uis.edu.co

2 Doctor en Administración de Empresas. Profesor visitante de Warrington College of Business Administration, Universidad de la Florida, Estados Unidos. Correo electrónico: roberto.stein@warrington.ufl.edu

3 Doctor en Administración. Profesor titular de la Escuela de Estudios Industriales y Empresariales de la Universidad Industrial de Santander, Bucaramanga, Colombia. Correo electrónico: cvecino@uis.edu.co 


\section{Introducción}

Entre los grandes problemas presentes en las finanzas, se encuentra la necesidad de perfeccionar los sistemas de predicción de comportamientos futuros en aras de tomar las mejores decisiones. Sin embargo, en el mejor de los casos se ha podido establecer que es a partir del análisis riguroso del comportamiento histórico como se podría llegar a una aproximación medianamente válida de pronósticos futuros.

Para el caso de la administración de portafolios de inversión, la aplicabilidad de dicho método es aún más tangible, debido a la existencia de grandes bases de datos históricas que permiten comprobaciones y simulaciones retrospectivas, las cuales validan o invalidan diversos caminos decisionales y revalúan estrategias de inversión de los administradores en la actualidad. Y para ello, los dos elementos por excelencia activadores de dichos modelos resolutivos han sido el riesgo asumido y el retorno obtenido.

De esta forma, el uso de herramientas de tipo cuantitativo se torna imprescindible para estos efectos y se apela a postulados como el de Markowitz (1952) y Sharpe (1966) para el diseño de un modelo de optimización, en el que se maximice la relación retorno ajustado por riesgo y en donde los datos de entrada sean accesibles para cualquier inversionista potencial, a través del levantamiento de la data histórica (precios diarios de cierre de las acciones en el mercado bursátil).

Se presupuesta entonces un modelo en donde, a partir de la conformación simulada de portafolios pasados, puedan ser evaluados sus comportamientos en términos de retornos eventualmente obtenidos y riesgos eventualmente asumidos; y a la vez comparados con algún indicador de base que permita arrojar conclusiones sobre el desempeño de dicho modelo, frente al de un modelo de administración puramente pasiva (para nuestro caso el IGBC).

En últimas, se pretende recabar evidencias que inciten a probar que la aplicación de postulados teóricos básicos pudieron haber logrado mejores comportamientos, que los métodos de decisión que efectivamente se llevaron a cabo por aquellos administradores que siguieron el patrón del mercado a través de la replicación de la estructura del índice bursátil respectivo. Todo esto, claramente en la vía de soportar con futuros estudios un modelo más robusto de inversión, aplicable a diversos escenarios y bajo condiciones que afinen los resultados al más adecuado nivel posible.

Sin la posibilidad de comparación directa por la diferencia metodológica y ventana de tiempo estudiada, este experimento representa en primera instancia, la extrapolación para Colombia del trabajo de Lizama y Ciudad (2013), quienes aplicaron un modelo similar en el mercado chileno y llegaron a conclusiones interesantes en las que (entre otros resultados) se validó la presunción expuesta 
anteriormente para esa realidad. Para el caso del presente estudio, los resultados en Colombia se dan en la misma vía y sugiere su potencial teórico y práctico para efectos de aplicación en otros contextos.

El modelo de optimización utilizado para este propósito procesó datos históricos del mercado bursátil colombiano (de diciembre del 2001 a diciembre del 2012) y proporcionó un conjunto de ponderaciones, los cuales dieron origen a una estrategia de inversión que fue analizada y comparada con el Índice General de la Bolsa de Valores de Colombia (en adelante IGBC), logrando demostrar la efectividad del modelo.

El artículo está organizado de la siguiente forma: en primera instancia se hace un breve marco teórico de los postulados y criterios asumidos, se aborda un marco de antecedentes puntual para la realidad colombiana, se procede a describir la metodología utilizada en el estudio, luego se enuncian los resultados obtenidos para que finalmente se de paso a la discusión y a las conclusiones de rigor.

\section{Marco teórico}

Para el presente estudio se hizo uso del modelo de selección de portafolio de Markowitz (1952), sirviéndose de la medición de desempeño ajustada a riesgo, propuesta por Sharpe (1964).

\section{EI Modelo de selección de portafolio de Markowitz}

El concepto de diversificación existe desde hace mucho antes que la Teoría Moderna de Finanzas. En 1952 Harry Markowitz publicó de manera formal un modelo de selección de portafolio que incorporó los principios de diversificación. $\mathrm{Su}$ modelo fue el primer paso de la gestión de portafolio: la identificación de un conjunto de portafolios eficientes, o la frontera eficiente de activos riesgosos. La idea principal de este conjunto de portafolios riesgosos es que, para cualquier nivel de riesgo, interesa solo el portafolio con el mayor retorno esperado. Alternativamente la frontera es el conjunto de portafolios que minimiza la varianza para cualquier meta de retorno esperado.

La premisa de este modelo consiste en que se puede generalizar el problema de construcción de portafolio al caso de muchas acciones riesgosas y un activo libre de riesgo.

De esta forma, su aplicación consta de tres partes. Primero, se identifican las combinaciones de riesgo-retorno disponibles a partir de un conjunto de activos riesgosos; seguido de esto, se identifica el portafolio óptimo de activos riesgosos al encontrar los ponderadores del portafolio. Finalmente se escoge un portafolio completo al mezclar activos libres de riesgo con el portafolio riesgoso óptimo. 
Su aplicación corresponde, en primera instancia, a determinar las oportunidades de riesgo-retorno disponibles para el inversionista, que están resumidas en la frontera de mínima varianza de activos riesgosos. Esta concuerda con la varianza mínima posible que puede ser obtenida dado el retorno esperado de un portafolio. A partir de los datos de retornos esperados, varianza y covarianzas, se puede calcular el portafolio de mínima varianza para cualquier nivel de retorno esperado.

Todos los portafolios que se encuentran en la frontera de mínima varianza, a partir del portafolio global de varianza mínima hacia valores superiores, proporcionan las mejores combinaciones riesgo-retorno y por tanto son candidatos al portafolio óptimo. Otras opciones son consideradas ineficientes.

La segunda parte del plan de optimización incorpora al activo libre de riesgo. Para esto se busca la denominada CAL (Capital Asset Line) que se ubica sobre la frontera mencionada y que cuenta con el más alto ratio de rentabilidad-riesgo.

La CAL (Capital Asset Line) según el portafolio óptimo $P$ sería, en términos gráficos, la tangente a la frontera eficiente con mayor valor de su pendiente. Esta CAL supera a todas las alternativas posibles, por lo tanto el portafolio $P$ es un portafolio riesgoso óptimo.

Finalmente, el inversionista individual elige una mezcla apropiada entre el portafolio riesgoso óptimo $P$ y activos libres de riesgo.

Dado todo lo anterior, se debe considerar que Markowitz desarrolla su modelo sobre la base de los siguientes supuestos:

- El rendimiento de cualquier activo o portafolio es descrito por una variable aleatoria subjetiva, cuya distribución de probabilidad para el periodo de referencia es conocida por el inversor.

- El riesgo de un activo o portafolio viene medido por la varianza (o desviación típica) de la variable aleatoria representativa de su rendimiento.

- El inversor preferirá aquellos activos financieros que tengan un mayor rendimiento para un riesgo dado, o un menor riesgo para un rendimiento conocido. Esta regla de decisión se denomina conducta racional del inversor, supone que los inversores son adversos al riesgo.

- No existen "imperfecciones" de mercado, tales como impuestos y costos de transacción.

- Considera la perfecta divisibilidad de los activos seleccionados, es decir, se pueden comprar en cualquier cantidad, incluso fracciones de ellos mismos.

El procedimiento de Markowitz presenta dos problemas. El primero, el modelo requiere una gran cantidad de estimadores para completar la matriz de covarianza. 
El segundo, no provee una guía para la proyección de las primas por riesgo de las acciones que son esenciales para construir la frontera eficiente de activos riesgosos.

Es por esto que se presenta un modelo de índice (Single-Index Model o SIM) que simplifica la estimación de la matriz de covarianza y mejora considerablemente el análisis de primas por riesgo de las acciones. Al permitir descomponer el riesgo en su componente sistemático y no sistemático (específico de la empresa), estos modelos también dejan de manifiesto el poder y las limitaciones de la diversificación. Además, permiten medir estos componentes del riesgo para una acción en particular o para un portafolio.

El evaluar el desempeño basándose solamente en el retorno obtenido, no es algo muy útil. Los retornos deben ajustarse por riesgo para que de esta manera puedan ser comparados significativamente.

Los métodos de desempeño ajustados por riesgo usando el criterio media-varianza surgieron simultáneamente con el CAPM. Los mismos Sharpe (1966), Treynor (1965) y Jensen (1968) reconocieron las implicancias del CAPM para elaborar un rating de desempeño de las administraciones. En este sentido, el desempeño de un portafolio es resultado de las decisiones que toma el administrador, entre ellas la elección de los activos. En últimas, evaluar el desempeño revela las habilidades y valor agregado del administrador.

\section{El ratio de Sharpe (SR)}

Es importante mencionar que los inversionistas presumiblemente están interesados en el retorno esperado en exceso por sobre el retorno libre de riesgo reemplazando dichos papeles con un portafolio riesgoso, como también en el riesgo que para esto incurren. Mientras la tasa libre de riesgo no es fija para cada periodo, se sabe con certeza qué tasa se obtendrá si este instrumento se compra y mantiene hasta su vencimiento.

Los inversionistas valoran los activos riesgosos de modo que el premio por riesgo sea acorde con el riesgo de ese exceso de retorno esperado, y por lo tanto, es mejor medir el riesgo mediante la desviación estándar del exceso de retorno, no sobre el total.

La importancia del trade-off entre la recompensa (premio por riesgo) y riesgo (medido mediante la desviación estándar) sugiere que se mida el atractivo de un portafolio de inversión mediante el ratio de su premio por riesgo a su desviación estándar de su exceso de retorno de modo que:

$$
S R=\frac{R_{p}-r_{f}}{\sigma\left(R_{p}-r_{f}\right)}
$$


Donde,

$R_{p}=$ Rentabilidad del portafolio

$r_{f}=$ Rentabilidad libre de riesgo

$\sigma\left(R_{p}-r_{f}\right)=$ Desviación estándar de la rentabilidad en exceso del portafolio

El SR se ha convertido en un estándar en la industria. Ha sido muy popular debido a que es una medida simple de calcular y que puede ser usada para comparar con relativa facilidad distintos tipos de estrategias de inversión. A pesar de lo anterior, es importante señalar que no es perfecto y en relación con sus debilidades y principales críticas es posible comentar que:

- No toma en cuenta si la volatilidad es al alza o a la baja, tratando todo tipo de volatilidad de la misma manera. Es decir, que un sistema cuyas ganancias tengan mucha desviación unas de otras, resultará en un SR pobre aunque la estrategia sea mejor que otra más "constante". Para un inversionista que busca potenciales estrategias de inversión, una volatilidad al alza no es necesariamente algo malo, sin embargo, el SR no lo diferencia y por tanto la volatilidad es penalizada en la fórmula. Esto potencialmente podría llevar a pensar al inversionista que la inversión no resulte atractiva, debido a que el ratio ve de igual forma volatilidad negativa y positiva.

- Un SR negativo indica que la estrategia o activo, siendo analizado, tiene menor desempeño que la tasa libre de riesgo. Manteniendo constante el resto de las variables, el ratio del portafolio debería disminuir si se incrementa el riesgo. Al mirar la formula, se puede apreciar que esto es cierto solo cuanto el SR es positivo. Por tanto, con un SR negativo, incrementar el riesgo resulta en un ratio más alto. Se concluye, que el SR no debe ser utilizado como una medida para comparar portafolios que tienen un valor negativo en su cálculo.

El SR considera solo la desviación estándar como medida de riesgo. Esto puede ser problemático cuando se calcule para activos con retornos asimétricos, debido a que la desviación estándar es apropiada como medida de riesgo de estrategias con una distribución de los retornos aproximadamente simétrica.

\section{Marco de antecedentes y coyuntura}

Los análisis que en la literatura se encuentran sobre el comportamiento histórico o retrospectivo del mercado de valores en Colombia, especialmente con lo que tiene que ver con el estudio del IGBC se limitan principalmente a diagnósticos estadísticos que en algunos casos demuestran su no-linealidad en media (Ospina \& Caicedo, 2008) y en otros (Alonso \& García, 2009) validan la existencia de 
ciertos patrones comportamentales, teniendo en cuenta el efecto Leverage, el efecto Día de la Semana y el efecto Hora, entre otros.

A pesar de esto, y de acuerdo con el estudio de Rivera (2009) se llega a la conclusión de que con la evidencia del impacto de días particulares sobre el cambio de los precios de las acciones, los resultados demuestran que los mercados de valores en Colombia no han sido eficientes en información; es decir, que los precios de las acciones no reflejan su valor verdadero y en consecuencia, el mercado está altamente expuesto a movimientos especulativos.

Recientemente, Fernández (2010) estudió la dinámica del IGBC y de su volatilidad. Los resultados que se obtienen sugieren que el modelo EGARCH puede ser mejor que el modelo GARCH para capturar los hechos estilizados del comportamiento del mercado colombiano. De la misma forma, se evidenció la importancia de los modelos asimétricos para estimar la volatilidad de series.

También se vislumbran algunos intentos de estimación de comportamientos futuros. Algunos de ellos basan su análisis en modelos como el ARCH o el Modelo Empírico de Tiempo Continuo; que de acuerdo con Grajales y Pérez (2008) son razonablemente adecuados para estimar la volatilidad del índice en mención, con el potencial agregado de determinación de la evaluación temporal de la distribución de volatilidad y subsecuentemente en la valoración de derivados sobre dichos índices.

Así mismo, se encuentra una sofisticación en el análisis con la utilización, por parte de Londoño, Lopera y Restrepo (2010), de redes neuronales artificiales, en donde además de la efectividad del método sobre los modelos estadísticos tradicionales, también demuestran la efectividad del modelo APT convencional (basado en datos fundamentales macroeconómicos) para predecir el comportamiento futuro del mercado de valores colombiano.

Por su parte, Alonso y Torres (2014) continuaron el trabajo de Alonso y Arcos (2006) y a través de la utilización de una batería amplia de pruebas estadísticas pudieron documentar la presencia de cinco hechos estilizados en el comportamiento de los rendimientos del IGBC en sus primeros 10 años (julio 2001 - julio 2011), estos son: 1) No se presenta eficiencia suave del mercado; 2) Colas pesadas de la distribución de los rendimientos; 3) Normalidad agregada de la distribución de los rendimientos, 4) Volatilidad no constante y agrupada de los rendimientos y, 5) Efecto Taylor.

De cualquier forma, algunos aportes relevantes que vale la pena mencionar tienen que ver con estudios exploratorios relacionados con los conceptos básicos de la teoría de portafolio. Es el caso de Dubova (2005) y Vélez (2007) en donde básicamente se encontró que no es posible establecer una relación entre la prima de riesgo y los retornos esperados, debido a la ineficiencia del mercado accionario colombiano con respecto a la información. Resultados que son compatibles con 
las conclusiones de Duarte, Ramírez y Mascareñas (2013) quienes adicionalmente demostraron la no existencia de una prima de riesgo adicional debida al tamaño de las empresas, rechazando tanto el Efecto Tamaño como el Efecto Invertido en el mercado de valores colombiano.

Sin embargo, más allá de contar con una extensa bibliografía que estudia y analiza el mercado en su integralidad, no se evidencian en la literatura estudios concretos que simulen el comportamiento de eventuales portafolios en condiciones reales a través de metodologías retroactivas, por lo que el análisis propuesto en la presente investigación se constituye como de carácter inédito y susceptible de desarrollo futuro a profundidad en aras de llegar a ser propositivo para la real toma de decisiones de inversión de los administradores.

Así mismo, es pertinente detenerse un poco en la descripción previa de un par de hechos específicos con respecto al periodo en que se proyectó la simulación respectiva, ya que de antemano se podrá percibir de manera muy lógica, un muy débil desempeño del mercado bursátil colombiano en esa ventana de tiempo de seis años, al observar el comportamiento de su índice accionario (que en términos totales alcanzó a avanzar solo un 31,85\%). Ellas explican en gran parte la situación, y en su mayoría coinciden con su presión sobre otros fenómenos similares en mercados accionarios de la región y del mundo.

En primera instancia, es necesario comentar que junto con situaciones internas de índole diplomático, la denominada crisis de las hipotecas sub-prime de Estados Unidos hizo que los años 2007 y 2008 fueran regresivos en su efecto directo hacia el mercado de valores colombiano, que implicó una fuerte desvalorización accionaria como consecuencia de los altos niveles de riesgo que se presentaban en el mercado. De esta misma forma, existió otra presión (esta vez no tan pronunciada) hacia la baja durante el 2011, como producto de la crisis de deuda de los países de la Unión Europea, debido a su cohesión monetaria.

\section{Metodología}

Se realizó una prueba retrospectiva de una estrategia de inversión basada en la construcción de portafolios óptimos, a través de la maximización del ratio de Sharpe, y de un algoritmo que como solución óptima genera un conjunto de ponderadores óptimos de inversión, que maximizan la relación retorno esperadoajustado por riesgo.

Como se comentó anteriormente, se decide utilizar en este estudio el modelo estadístico de valoración de activos, Single Index Model (SIM), debido a que especifica que todos los movimientos comunes entre acciones pueden ser capturados por un único índice, en este caso particular el IGBC. Además de ser un modelo 
simplificador del Modelo de Selección de Portafolio de Markowitz, al reducir el número de datos requeridos para su uso.

\section{Selección de activos para la construcción de portafolios}

Para el desarrollo del modelo de optimización mencionado anteriormente, es necesario considerar algunos criterios que se han definido de manera para obtener una base de datos confiable y debidamente estructurada:

- La información utilizada para la optimización del portafolio considera un índice representativo del mercado colombiano de renta variable, las 32 acciones pertenecientes al IGBC vigente para el 2012.

- La muestra utilizada para la optimización es de frecuencia diaria, se utilizan precios de cierre (ajustados para reflejar escisiones, canjes o consolidaciones de acciones, dividendo/incentivo en acciones y ofertas de derecho), obtenidos a través del terminal de información internacional Bloomberg (fórmula histórica para bajar a Excel los datos de una acción = BDH ("Ticker acción", "PX_LAST", "Fecha inicial", "Fecha final"), a través de los cuales se obtienen retornos diarios de acuerdo con la fórmula de media aritmética:

$$
R_{t}=\frac{\left(P_{t}-P_{t \rightarrow 1}\right)}{P_{t \rightarrow 1}}
$$

Donde $P_{t}$ corresponde al Precio final de la acción, y $P_{t \rightarrow 1}$ al Precio inicial de la acción.

El algoritmo de optimización considera solo acciones que tengan por lo menos cinco años de data histórica, de este modo se eliminan aquellas que tengan datos N/A. A su vez, de las acciones del IGBC se excluyen aquellas que hayan presentado al menos un mes sin variación de precio (retorno mensual $=0 \%$ ) con el objetivo principal de no considerar aquellas acciones que pudieran presentar problemas de liquidez por falta de transacción.

En la búsqueda de simplificar este estudio, adicional a los supuestos propios del modelo de Markowitz, se considera que:

- Todos los inversionistas pueden acceder a la misma tasa libre de riesgo.

- La información es gratuita, instantánea y disponible para todos los inversionistas.

- No se permite la venta corta. Solo se consideran posiciones de compra para las acciones seleccionadas por el modelo de optimización diseñado. 
Así mismo, a pesar de haber hecho una mención anteriormente, no se tiene en cuenta el comportamiento particular de los fenómenos del entorno en términos de puntos críticos (apogeos y crisis económicas acontecidas) de los valores, ya que, adicional a la convencionalidad de estas circunstancias y al tamaño considerable de la ventana de tiempo analizada, la comparación que se plantea con el IGBC también permite pensar que la exposición de todas las referencias ha sido la misma y por lo tanto las conclusiones del ejercicio no son afectadas por dichas situaciones.

\section{Resultados}

A continuación se exponen y analizan los resultados obtenidos de la aplicación del modelo de optimización del portafolio, mediante la maximización del ratio de Sharpe, en adelante indistintamente modelo de optimización o algoritmo.

\section{Estrategia de inversión y criterios de rebalanceo}

En este estudio se propone y analiza una estrategia de inversión en renta variable, la cual se crea a partir de una ventana inicial de análisis de cinco años, una ventana móvil de cinco años y un horizonte de inversión de seis años, es decir, seis rebalanceos y cambios consecutivos en la composición del portafolio.

Descripción de la simulación: se invierte (inicio 31/12/2006) un monto de dinero específico en un portafolio óptimo, cuyas acciones y ponderación de inversión por acción se determina a través de la optimización del SR, aplicada a retornos accionarios históricos de un periodo precedente de cinco años (20022006). Se mantiene esta inversión por el periodo de un año, luego del cual, se reinvierte nuevamente por el mismo periodo, el monto de inversión más la ganancia obtenida en un nuevo portafolio que es determinado a través de la optimización del SR aplicado a retornos accionarios históricos precedentes de la nueva ventana de tiempo de cinco años. Para su constitución se puede comprar, vender o mantener acciones y ponderación de inversión del portafolio anterior.

De esta manera, adicional a los portafolios creados para cada periodo, obtenemos los retornos alcanzados por su eventual inversión. Dichos datos son comparados con sus equivalentes reales del comportamiento del IGBC que corresponde al índice bursátil más representativo del mercado de valores colombiano y que está compuesto por un ponderado de las acciones más representativas en función de su liquidez y capitalización. De esta manera, su comportamiento es una radiografía directa del comportamiento del mercado bursátil de ese país, en términos generales, y la comparación realizada representa una emulación de comparación directa con el mercado per se.

Luego de procesar la simulación en lenguaje R (que se caracteriza por ser gratuito, libre y de código abierto) se destacó: 
El algoritmo para los seis años de inversión, de un total de 32 acciones comunes, entregó recomendaciones óptimas de solo siete acciones para los seis años de inversión; estas fueron Bcolo, Celsia, Corficol, Éxito, Gruposur, Mineros y Nutresa.

Los sectores económicos a los que pertenecen dichas acciones son cuatro: dos acciones correspondientes al sector industrial, dos acciones al sector financiero, dos al sector inversiones y solo una acción perteneciente al sector comercial (tabla 1).

Tabla 1. Nombre de las compañías recomendadas con sus sectores económicos

\begin{tabular}{|c|c|c|}
\hline Acción & Nombre & Sector \\
\hline BCOLO & Bancolombia S.A. & Financiero \\
\hline CELSIA & Celsia S.A. E.S.P. antes Colinvers & Industrial \\
\hline CORFICOL & Corporación Financiera Colombiana Corficolombiana & Financiero \\
\hline ÉXITO & Almacenes ÉXITO S.A. & Comercial \\
\hline GRUPOSUR & Grupo de Inversiones Suramericana S.A. & Inversiones \\
\hline MINEROS & Mineros S.A. & Industrial \\
\hline NUTRESA & Grupo Nutresa S.A. & Inversiones \\
\hline
\end{tabular}

Fuente: página web Bolsa de Valores de Colombia (www.bvc.com.co)

También es necesario mencionar que a pesar del aparente atractivo reciente de acciones como EEB y Ecopetrol; el modelo de optimización las excluyó de las recomendaciones respectivas, en gran parte debido a su "juventud" en el mercado bursátil colombiano, ya que iniciaron su cotización formal entre e 12007 y el 2011, lo cual impidió un análisis retroactivo más profundo por parte del algoritmo creado, y por ende su "referencia futura".

El algoritmo recomendó portafolios de al menos dos acciones para todos los años; excepto para el 2011 cuando recomendó una inversión total sobre la acción de Mineros; y en ningún caso se presentó recomendación de portafolio superior a tres acciones.

De acuerdo con lo anterior, se concluye que los portafolios sugeridos no fueron compatibles con el principio financiero básico de diversificación, que declara que un inversionista no debe colocar todos sus recursos en un solo activo $o$ en un número relativamente pequeño de activos, sino en un número grande de instrumentos de inversión, ayudando a amortiguar la variabilidad de los rendimientos de los activos individuales. La razón atribuible a este fenómeno es probablemente la poca profundización del mercado de valores colombiano $\mathrm{y}$, por consiguiente, la poca visibilidad que tienen algunas acciones que no han 
consolidado históricamente su atractivo en términos de rentabilidad o riesgo; de tal forma que el modelo no las tuvo en cuenta para sus recomendaciones.

La composición de los portafolios recomendados por la estrategia de inversión aplicada se puede apreciar en la figura 1.

Figura 1. Portafolios anuales

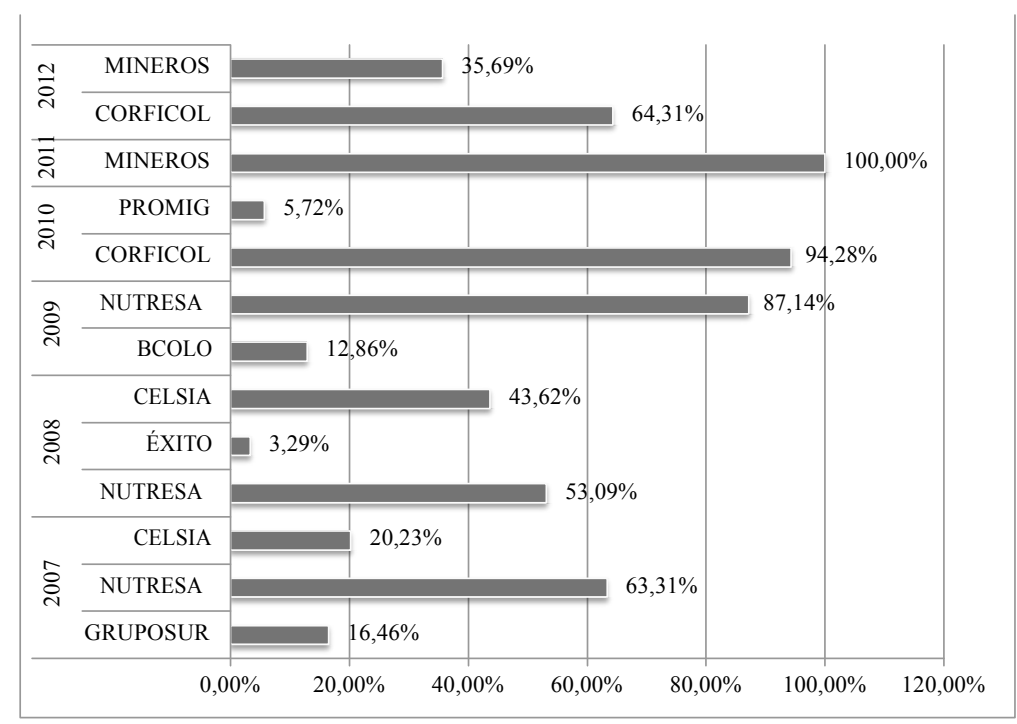

Fuente: autores

En cuanto al valor del portafolio total, en la figura 2 se puede apreciar el comportamiento simulado de una inversión para la estrategia de rebalanceo establecida durante el periodo 2007-2012, asumiendo una inversión de \$1 el día 31 de diciembre del 2006.

De esta forma se obtiene un valor de portafolio final de un $46,53 \%$ por encima del valor de portafolio inicial. En términos sencillos significa que si el 30 de diciembre del 2006 se hubiese invertido \$1 en un plazo de 6 años con un rebalanceo cada año de acuerdo con el modelo arrojado, se hubiese contado con $\$ 1,47$ el día 30 de diciembre del año 2012. 
Figura 2. Valor del portafolio recomendado vs. IGBC

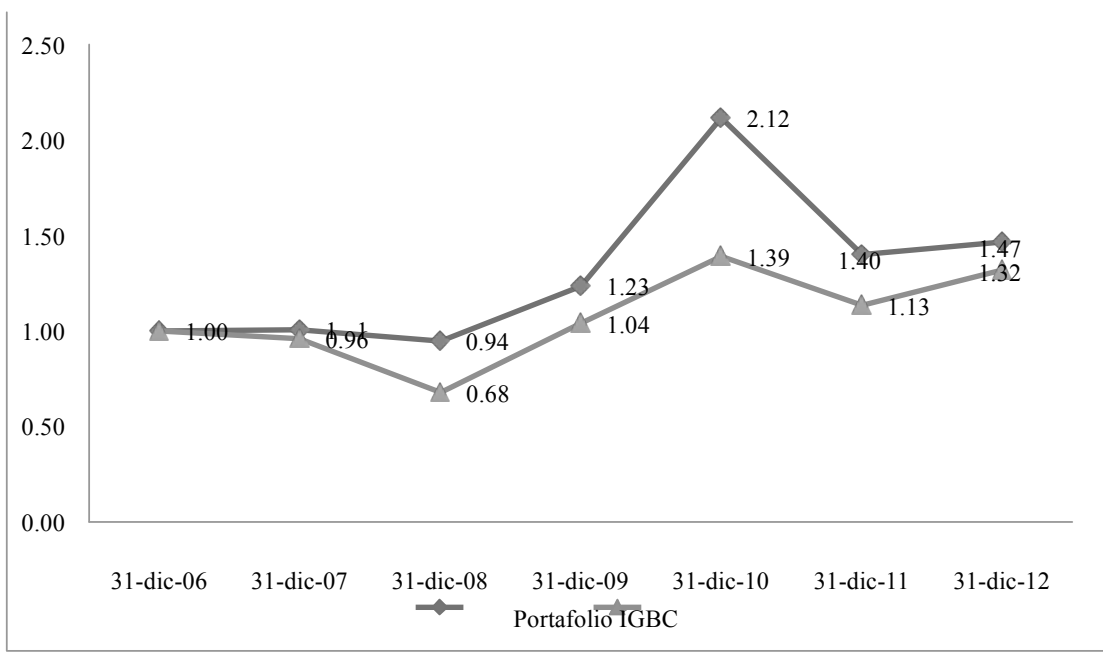

Fuente: autores.

En comparación con el IGBC, se puede observar claramente que el desempeño de los portafolios propuestos es sustancialmente mejor que el arrojado por el indicador mencionado, ya que desde su valor registrado el 30 de diciembre del 2006 (COP\$11.161,14), el IGBC ascendió a un valor de COP\$14.715,84 para el día 30 de diciembre del 2012. Lo que quiere decir que su valorización real fue de $31,85 \%$ durante los años 2007-2012; o en términos más simples, que si se hubiese invertido $\$ 1$ en un plazo de seis años a un activo hipotético denominado IGBC (con el comportamiento real del índice en mención, como ejemplo de un fondo de administración pasiva) mediante una estrategia en la cual se compra desde el inicio y se mantiene sin adición o resta hasta el final del periodo, se hubiese contado con \$1,32 al final del día 30 de diciembre del 2012.

Asimismo, al analizar cada uno de los periodos en términos de sus rendimientos puntuales (no valores) se evidencia, que, muy a pesar de comportamientos puntuales de interesante diferencia en contra de los portafolios propuestos (especialmente para el 2009, 2011 y 2012); al final también se logra un rendimiento promedio superior para la estrategia recomendada que valida una vez más la pertinencia del modelo en las condiciones planteadas (tabla 2). 
Tabla 2. Retornos anuales - Estrategia de inversión recomendada vs. IGBC

\begin{tabular}{|c|c|c|}
\hline Periodo & Ret. anual Portafolio & Ret. anual IGBC \\
\hline 2007 & $0.66 \%$ & $-4.18 \%$ \\
\hline 2008 & $-6.15 \%$ & $-29.30 \%$ \\
\hline 2009 & $30.60 \%$ & $53.45 \%$ \\
\hline 2010 & $71.48 \%$ & $33.57 \%$ \\
\hline 2011 & $-33.79 \%$ & $-18.27 \%$ \\
\hline 2012 & $4.60 \%$ & $16.19 \%$ \\
\hline Ret. anual promedio & $\mathbf{1 1 , 2 3} \%$ & $\mathbf{8 . 5 8 \%}$ \\
\hline
\end{tabular}

Fuente: autores

Desde otro punto de vista, en el cual se tiene en cuenta la tasa de interés obtenida capitalizable cada periodo (para este caso los periodos son los mismos años), se podría afirmar que un fondo pasivo indexado al IGBC obtuvo un rendimiento anual equivalente al $4.74 \%$ capitalizable año a año en el periodo comprendido entre 2002 y 2006, y que con la aplicación del modelo de optimización propuesto, que se basó en rebalanceos anuales de portafolios arrojados por la maximización del SR, durante la misma ventana de tiempo se hubiese obtenido un rendimiento anual equivalente al $6,57 \%$ capitalizable año a año (tabla 3 ).

Tabla 3. Evolución de una inversión de \$1 con rendimientos capitalizables año a año

\begin{tabular}{|c|c|c|}
\hline Fecha & \multicolumn{2}{|c|}{ Valor a la fecha } \\
\hline & Portafolio & IGBC \\
\hline 31-dic-06 & 1.00 & 1.00 \\
\hline 31-dic-07 & 1.07 & 1.05 \\
\hline 31-dic-08 & 1.14 & 1.10 \\
\hline 31-dic-09 & 1.21 & 1.15 \\
\hline 31-dic-10 & 1.29 & 1.20 \\
\hline 31-dic-11 & 1.37 & 1.26 \\
\hline 31-dic-12 & 1.47 & 1.32 \\
\hline Rendimiento capitalizable & $\mathbf{6 . 5 7 \%}$ & $\mathbf{4 . 7 4 \%}$ \\
\hline
\end{tabular}

Fuente: autores. 
Con respecto a estos análisis de confrontación con el IGBC, es necesario mencionar que tal como lo afirman Orito, Inoguchi, Yamamoto y Ronbunshi (2010) son ampliamente tratados en la literatura los casos de los sobresalientes comportamientos operacionales de los fondos de administración pasiva en comparación con otros tipos de fondos. Los experimentos de Malkiel (1995), Gruber (1996) y Elton, Gruber y Blake (1996), y más recientemente, Phillips, Kinniry, Schlanger y Hirt (2014) dan cuenta de dicha afirmación y la validan.

Así las cosas, de acuerdo con la comparación presentada en donde evidentemente se observa un mejor comportamiento por parte de los portafolios simulados frente al comportamiento del IGBC, se concluye que (bajo las condiciones asumidas) la aplicación del modelo de optimización hubiese podido constituirse en una herramienta efectiva para la administración activa de portafolios durante los periodos analizados; y que de acuerdo con los resultados obtenidos, podría ser útil su uso eventual en el presente para la constitución futura de portafolios de inversión en el mercado de valores colombiano.

\section{Conclusiones}

- La estrategia de inversión arrojada por el modelo de optimización desarrollado demuestra una constante superioridad en términos de su valor de portafolio, alcanzando un valor final para los seis años de inversión superior a los índices de mercado, representados en los valores reales del IGBC durante esa misma ventana de tiempo.

- También se arrojan evidencias de la superioridad del modelo en términos financieros, ya que para efectos de su análisis de retornos obtenidos, la misma estrategia arroja cifras por encima de los retornos reales de este mismo indicador, tanto en el rendimiento promedio como en el rendimiento capitalizable en los periodos estudiados.

Lo anterior conduce a pensar que de acuerdo con los análisis desarrollados y los resultados obtenidos, el uso de modelos de optimización basados en conceptos y herramientas tradicionales financieras es válido y efectivo en términos de administración de portafolios en Colombia. Y que incluso, podría llegar a ser más efectivo que los criterios utilizados actualmente por los administradores.

- Como todo algoritmo o modelo de optimización, el enfoque en la maximización del ratio de Sharpe tiene ciertas limitantes, como la de asumir la no existencia de costos de transacción e impuestos. Sin embargo, el impacto de incorporarlos en las estrategias de inversión no afecta mayormente sus resultados (en particular para montos de inversión considerables), ni las 
conclusiones ya mencionadas, ya que sus parámetros de comparación estarían actuando bajo las mismas condiciones y de una $\mathrm{u}$ otra forma su efecto sobre el análisis es mutuamente compensado. Sin embargo, futuros análisis podrían contemplar esta variable utilizando datos reales o calculando valores máximos permitidos que soporten o invaliden los resultados actuales.

- No fue posible el análisis comparativo entre el desempeño de los portafolios recomendados y la cotización del índice Colcap de la BVC, que aunque refleja las variaciones de los precios de las veinte acciones más líquidas del mercado de valores de ese país, su "reciente" debut bursátil acontecido el día 15 de enero de 2008, impide su inclusión en el análisis presente por el evidente impedimento de observación frente a la ventana de tiempo examinada.

- Por la relevancia de los resultados obtenidos y la existencia de antecedentes directos como lo es el trabajo de Lizama y Ciudad (2013) para el caso chileno, es claro el potencial de desarrollo de investigaciones más profundas en donde se evalúen diferentes ventanas de tiempo o estrategias de inversión, basadas en variedad de la frecuencia en los rebalanceos. Este tipo de trabajos futuros brindarán conclusiones más sólidas y aportes más concretos para incluso, soportar una implementación del modelo en entornos reales.

\section{Referencias}

Alonso, J. C. y Arcos, M .A. (2006). Cuatro hechos estilizados de las series de rendimientos: Una ilustración para Colombia. Estudios Gerenciales, 22(100), $103-123$

Alonso, J., \& García, J. (2009). ¿Qué tan buenos son los patrones del IGBC para predecir su comportamiento? Una aplicación con datos de alta frecuencia. Estudios Gerenciales, 25(112), 13-36.

Alonso, J., \& Torres, G. (2014). Características estadísticas del índice general de la Bolsa de Valores de Colombia (IGBC) en sus primeros 10 años. Journal of Economics, Finance and Administrative Science, (19), 45-54.

Duarte, J. B., Ramírez, Z. Y., \& Mascareñas, J. M. (2013). Estudio del efecto tamaño en el mercado bursátil colombiano. Journal of Economics Finance and Administrative Science, 18(Special Issue), 23-27.

Dubova, I. (2005). La validación y aplicabilidad de la teoría de portafolio en el caso colombiano. Cuadernos de Administración, 18(30), 241-279.

Elton, E. J., Gruber, M. J., \& Blake, C. R. (1996). Survivorship bias and mutual fund performance. The Review of Financial Studies, 9(4), 1097-1120. 
Fernández, H. (2010). Una aplicación del modelo EGARCH para estimar la volatilidad de series financieras. Revista Ingenierías Universidad de Medellín, 9 (17), 95-104.

Grajales, C., \& Pérez, F. (2008). Modelos discretos continuos para estimar la densidad de probabilidad de la volatilidad escolástica de los rendimientos de series financieras. Cuadernos de Administración, 21 (36), 113-132.

Gruber, M. J. (1996). Another Puzzle: The growth in actively managed mutual funds. The Journal of Finance, 51(3), 783-810.

Jensen, M. C. (1968). The performance of mutual funds in the period 1945-1964. The Journal of Finance, 23 (2), 389-416.

Lizama, C., \& Ciudad, P. (2013). Diseño y evaluación de estrategias óptimas para la selección de portafolios, mediante la maximización del ratio de Sharpe. (Tesis de maestría no publicada), Facultad de Economía y Negocios, Universidad de Chile. Santiago, Chile.

Londoño, C., Lopera, M., \& Restrepo, S. (2010). Teoría de precios de arbitraje. Evidencia empírica para Colombia a través de redes neuronales. Revista de Economía del Rosario, 13(1), 41-74.

Malkiel, B. G. (1995). Return from investing in equity mutual funds 1971-1991. Journal of Finance, 50(2), 549-572.

Markowitz, H. M. (1952). Portfolio Selection. The Journal of Finance, 7(1), 77-91.

Orito, Y., Inoguchi, M., Yamamoto, H., \& Ronbunshi, D. (2010). Index fund optimization using a genetic algorithm and a heuristic local search. Electronics and Communications in Japan, 93(10), 407-415.

Ospina, J., \& Caicedo, E. (2008). Un modelo estocástico sobre la predictibilidad del signo de retorno y su relación con la no linealidad en media. Cuadernos de Administración, 21(36), 11-35.

Phillips, C. B., Kinniry Jr., F. M., Schlanger, T., \& Hirt, J. M. (2014). The case for index- fund investing. Vanguard. Retrieved 2 january de 2014 From:https:// pressroom.vanguard.com/content/nonindexed/Updated_The_Case_for_In dex Fund_Investing_4.9.2014.pdf

Rivera, D. (2009). Modelación del efecto del día de la semana para los índices accionarios de Colombia mediante un modelo Star Garch. Revista de Economía del Rosario, 12(1), 1-24.

Sharpe, W. (1964). Capital asset prices: A theory of market equilibrium under conditions of risk. The Journal of Finance, XIX(3), 425-442.

Sharpe, W. (1966). Mutual Fund Performance. Journal of Business, 39(1), 119-138. 
Treynor, J. L. (1965). How to rate management of investment funds. Harvard Business Review, 43(1), 63-75.

Treynor, J. L., \& Black, F. (1973). How to use security analysis to improve portfolio selection. Journal of Business, 46(1), 66-86.

Vélez, J. (diciembre, 2007). CAPM: Teoría y hallazgos empiricos para Colombia, 2001-2006. Simposio Internacional de Finanzas. Conferencia realizada en la Pontificia Universidad Javeriana, Cartagena, Colombia. 